\title{
COMPRENDRE..
}

\section{Les capteurs à réseaux de Bragg}

\author{
Lionel QUETEL, \\ Patrice LEBOUDEC \\ IDIL Fibres Optiques, \\ 21 rue Louis De Broglie, \\ 22300 Lannion \\ lionel.quetel@idil.fr
}

\section{Le réseau de Bragg}

\section{Avant-propos}

Les méthodes d'inscription des réseaux de Bragg ont été ces dernières années considérablement améliorées si bien que le champ d'utilisation de ces composants s'est fortementétendu dans les domaines des télécommunications optiques et des capteurs à fibre. L'amélioration a tout d'abord porté sur une meilleure compréhension des mécanismes à l'origine de la photosensibilité des fibres germano silicates et sur l'établissement des lois qui régissent la cinétique d'inscription des réseaux. Sur un plan pratique, de nouvelles techniques d'inscription et de caractérisation de réseaux ont été proposées afin de s'affranchir en partie de
Les avancées technologiques récentes ont rendu possible l'utilisation des réseaux de Bragg à grande échelle dans le domaine des télécoms et, depuis une dizaine d'années, dans le domaine des capteurs. Ces capteurs à réseaux de Bragg se sont d'abord développés dans les secteurs du génie civil, de l'offshore et de l'aviation, et diffusent désormais dans des applications plus grand public, telles que l'automobile. certaines difficultés inhérentes àl'utilisation de la méthode d'holographie transverse initialement utilisée. Ainsi, grâce à l'utilisation de masque de phase, il est devenu possible d'inscrire les réseaux de façon reproductible. Les caractéristiques de ces réseaux peuvent être ajustées en fonction du type d'application visée.

\section{Le principe du réseau de Bragg}

Le réseau de Bragg est une modification permanente de l'indice de réfraction du cour d'une fibre optique obtenue par l'insolation transverse d'une figure d'interférence ultraviolette. La modulation de l'indice de réfraction ainsi obtenue constitue un

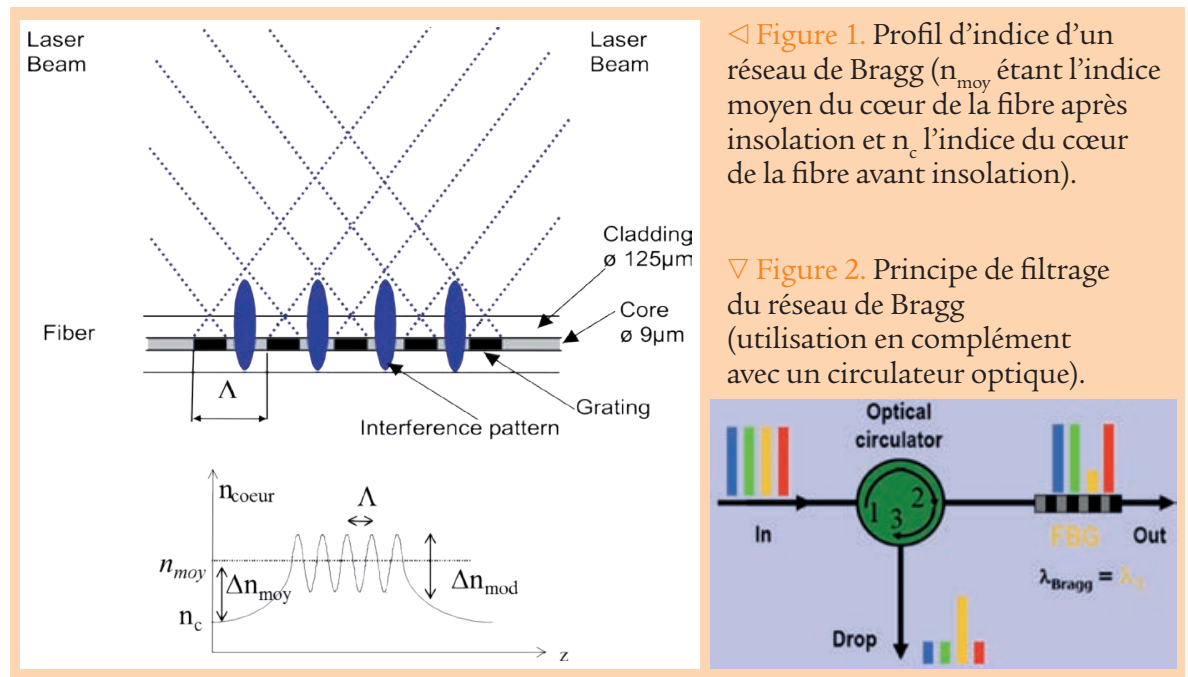

réflecteur sélectif en longueur d'onde. La longueur d'onde de réflexion est donnée par la relation:

$$
\lambda_{B}=2 n_{\text {eff }} \Lambda
$$

où $\lambda_{B}$ est la longueur d'onde de Bragg, $n_{\text {eff }}$ l'indice du mode guidé dans la fibre et $\Lambda$ le pas du réseau. La largeur spectrale du réseau dépend de la longueur physique du réseau: plus le réseau est long, plus sa largeur spectrale est faible. Typiquement un réseau de $10 \mathrm{~mm}$ de long possède une largeur spectrale de l'ordre de $0,1 \mathrm{~nm}$. La réflexion du réseau est fonction de la profondeur de modulation d'indice $\Delta n_{\text {mod }}$ et donc du temps d'insolation de la fibre ou de la puissance UV reçue par celle-ci. Les réflexions obtenues sont comprises entre quelques pourcents et $99,999 \%$.

Si l'on injecte une source ayant une large bande spectrale dans un réseau de Bragg, celui-ci va réfléchir une partie du signal à la longueur d'onde $\lambda_{\mathrm{B}}$ et va transmettre le reste des longueurs d'onde comme représenté en figure 1. Un coupleur ou un circulateur permettent de récupérer le signal réfléchi.

\section{Les moyens de photoinscription}

Plusieurs méthodes autorisent la photoinscription des réseaux de Bragg. Les deux méthodes les plus répandues sont décrites ci-dessous. 


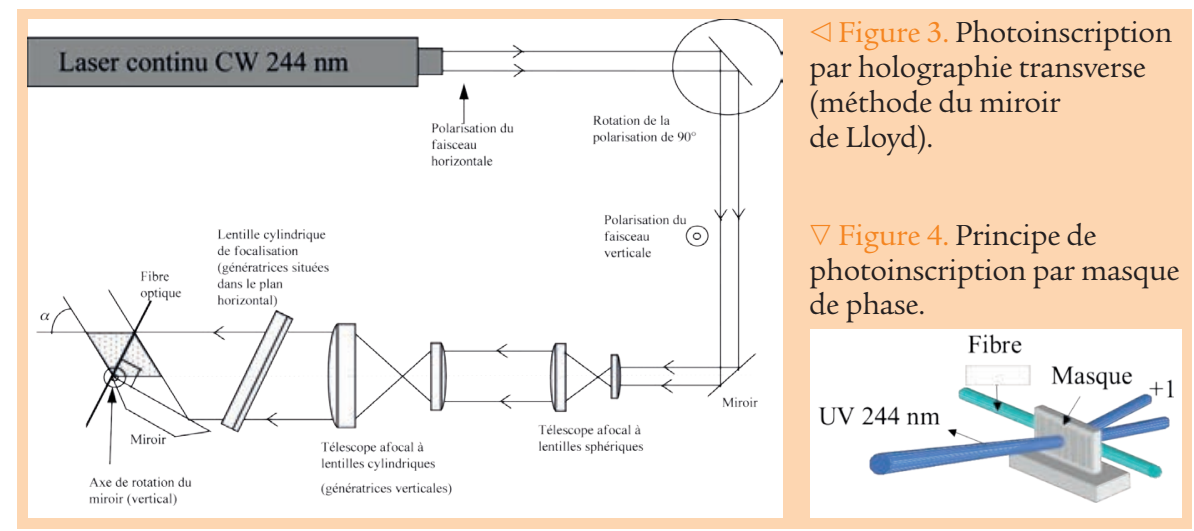

\section{Photoinscription par holographie transverse}

Un laser continu émettant à $244 \mathrm{~nm}$ et de grande longueur de cohérence est utilisé. Un ensemble de télescopes afocaux permet de réduire la divergence angulaire du faisceau et d'en augmenter la stabilité angulaire. Un miroir plan est disposé selon un plan vertical. Ce miroir est placé sur une platine de rotation. La fibre optique préalablement dénudée est disposée perpendiculairement au plan du miroir. Une lentille cylindrique permet d'augmenter l'éclairement du faisceau ultraviolet au niveau de la fibre optique. Le miroir joue le rôle d'un séparateur de front d'onde et provoque l'interférence entre le front d'onde réfléchi et le front d'onde transmis.

L'angle $\alpha$ entre les deux plans d'onde détermine la longueur d'onde de Bragg $\lambda_{B}$ du réseau. En effet, la longueur d'onde est donnée par la relation ci-dessous:

$$
\lambda_{B}=n_{\text {eff }} \frac{\lambda_{P}}{\sin \left(\frac{\alpha}{2}\right)}
$$

Dans cette relation, $n_{\text {eff }}$ désigne l'indice effectif du mode guidé pour la longueur d'onde de Bragg, $\lambda_{p}$ est la longueur d'onde du laser source (ici $244 \mathrm{~nm}$ ). La longueur d'onde de Bragg du réseau peut donc être modifiée par rotation du miroir rendant le procédé très flexible, et autorise la réalisation de réseaux de longueur voisine de quelques millimètres limités par la longueur de cohérence du laser UV. Mais cette méthode interférométrique est très sensible aux instabilités mécaniques et rend le procédé peu reproductible et donc difficilement compatible avec l'industrie.

\section{Photoinscription par masque de phase}

De par son principe de fonctionnement, l'utilisation d'un masque de phase permet de s'affranchir des limitations imposées par la longueur de cohérence des lasers utilisés. Le masque est constitué d'une lame de silice à faces parallèles sur laquelle ont été gravés des traits. Le faisceau ultraviolet traverse la lame de silice et se trouve diffracté par les traits du masque. Les ondes diffractées dans les ordres +1 et -1 créent un champ de franges d'interférence stable dont les plans d'égales intensités sont perpendiculaires au plan du masque comme représenté à la figure 4 .

La fibre optique dénudée est placée à une distance de l'ordre de $100 \mu \mathrm{m}$ du masque. Le pas de la modulation d'indice est égal à la largeur des traits gravés dans le masque de phase.

\section{Le réseau de Bragg capteur}

La longueur d'onde de Bragg étant donnée par la relation $\lambda_{B}=2 n_{\text {eff }} \Lambda$, l'indice effectif $n_{\text {eff }}$ et le pas physique du réseau étant dépendants de la température et de l'élongation de la fibre, la longueur d'onde de Bragg s'en retrouve aussi dépendante.

\section{La dépendance avec la température}

La dépendance de la longueur d'onde avec la température est donnée par la relation suivante:

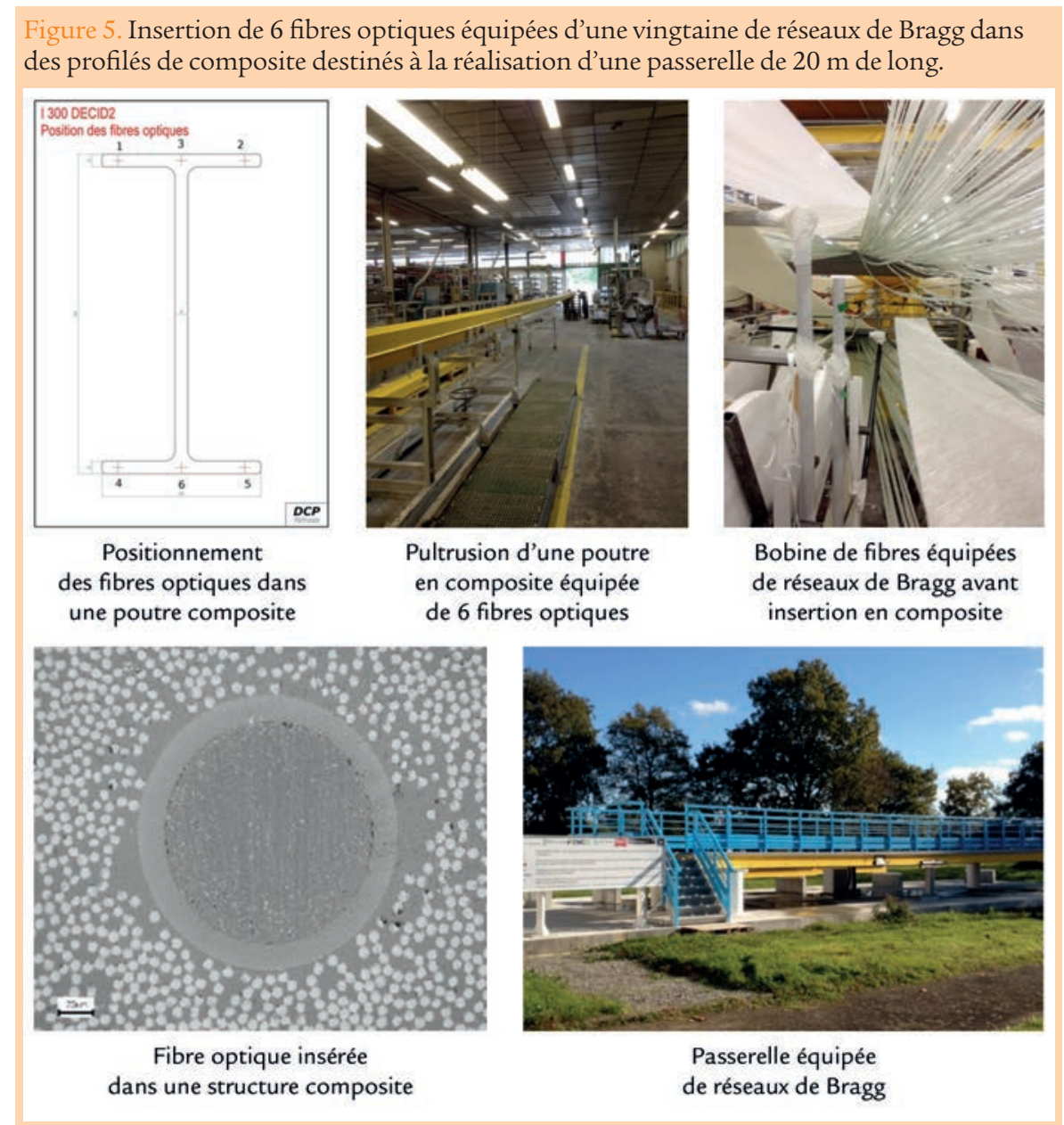




$$
\frac{\Delta \lambda_{B}}{\lambda_{B}}=(\alpha+\zeta) \Delta T
$$

$\Delta \lambda_{B}$ est la variation de la longueur d'onde de Bragg, $\alpha$ le coefficient de dilatation de la fibre, $\zeta$ le coefficient thermo-optique de la silice (habituellement dopée avec du germanium) et $\Delta T$ la variation de température. Les coefficients $\alpha$ et $\zeta$ sont définis par les relations:

$$
\alpha=\frac{1}{L} \frac{\partial L}{\partial T} ; \xi=\frac{1}{n_{e f f}} \frac{\partial n_{e f f}}{\partial T}
$$

où $L$ est la longueur du réseau, et $n_{\text {eff }}$ l'indice effectif. Dans la pratique, on suppose que les valeurs numériques du coefficient de dilatation et du coefficient thermo-optique d'une fibre photoinscrite sont sensiblement identiques à celles d'une fibre en silice pure, c'est-à-dire $\alpha=0,5510^{-6} \mathrm{~K}^{-1}$ et $\zeta=10^{-6} \mathrm{~K}^{-1}$. Nous pouvons admettre que $\lambda_{B}$ varie linéairement avec la température si nous supposons que $\alpha$ et $\zeta$ sont indépendants de $T$. La variation de la longueur d'onde de Bragg devient alors de $0,01 \mathrm{~nm} \mathrm{~K}^{-1}$ soit $10 \mathrm{pm} \mathrm{K}^{-1}$.

\section{La dépendance avec l'élongation}

Lorsqu'une force est appliquée longitudinalement sur une fibre optique, la fibre de longueur $L$ subit un allongement $\Delta L$ en raison de son élasticité. L'indice des matériaux constituant la fibre se trouve modifié par effet

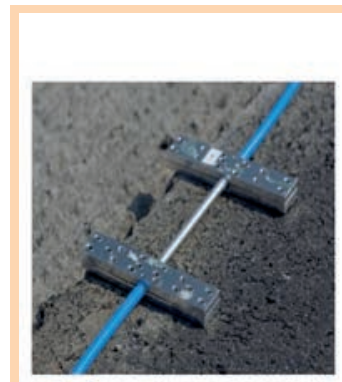

Réseau de Bragg conditionné pour insertion dans l'enrobé

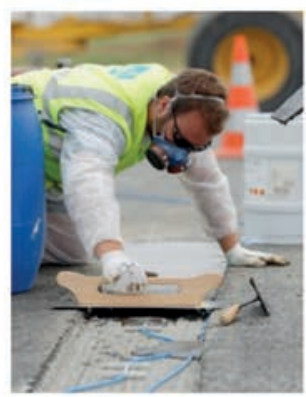

Insertion d'une chaîne de 16 réseaux de Bragg

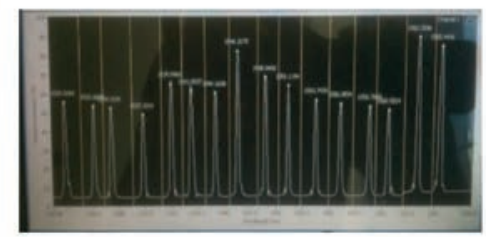

Spectre en réflexion de la chaîne de mesure

Figure 6. Conditionnement spécifique de réseau de Bragg pour insertion dans l'enrobé de tarmac.

photo-élastique. Dans le cas particulier d'une déformation axiale $\varepsilon\left(\varepsilon=\frac{\Delta L}{L}\right)$, la variation d'indice effectif du mode fondamental est isotrope et peut se déduire de la relation approchée:

$$
\Delta n_{\text {eff }}=-\frac{1}{2} n_{\text {eff }}^{3}\left[p_{12}-v\left(p_{11}+p_{12}\right)\right] \varepsilon
$$

Dans la relation ci-dessus, $n_{\text {eff }}$ est l'indice effectif, $p_{i j}$ sont les coefficients élasto-optiques et $v$ le coefficient de Poisson. En utilisant les relations: $\lambda_{B}=2 n_{\text {eff }} \Lambda$ et $\varepsilon=\frac{\Delta L}{L}$, la variation de la longueur d'onde de Bragg se déduit de la relation:

$$
\frac{\Delta \lambda_{B}}{\lambda_{B}}=\left(1-p_{e}\right) \frac{\Delta L}{L}
$$

où $p_{e}=\frac{n_{e f f}^{2}}{2}\left[p_{12}-v\left(p_{11}+p_{12}\right)\right]$. La valeur numérique de $p_{e}$ est proche de 0,22 pour des fibres en silice pure.

$$
\frac{\Delta \lambda_{B}}{\lambda_{B}}=0,78 \frac{\Delta L}{L}
$$

Silalongueurd'ondedetravailest $1,5 \mu \mathrm{m}$, on trouve alors $\Delta \lambda_{B}(\mathrm{pm})=1,2 \Delta L\left(\mu \mathrm{def}^{1}\right)$.

\section{Quelques exemples d'application}

Ce paragraphe énumère quelques exemples de mesures réalisées avec des réseaux de Bragg.

\section{Les réseaux de Bragg pour le génie civil}

Les capteurs à fibre optique (CFO) sont utilisés pour l'instrumentation des infrastructures de génie civil depuis près de trois décennies. Ce type de capteurs

$\mu$ def $=1$ déplacement de $1 \mu \mathrm{m}$ pour $1 \mathrm{~m}$.

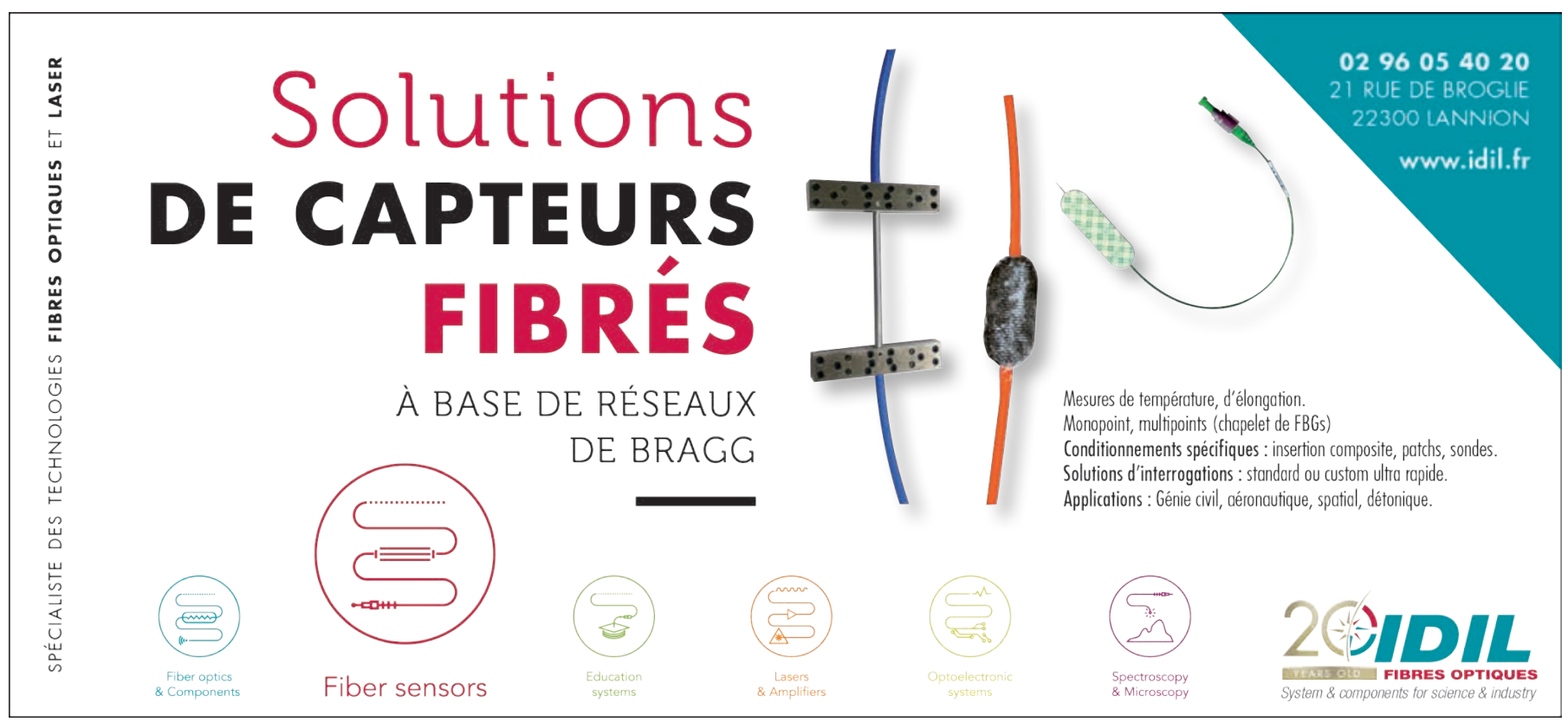




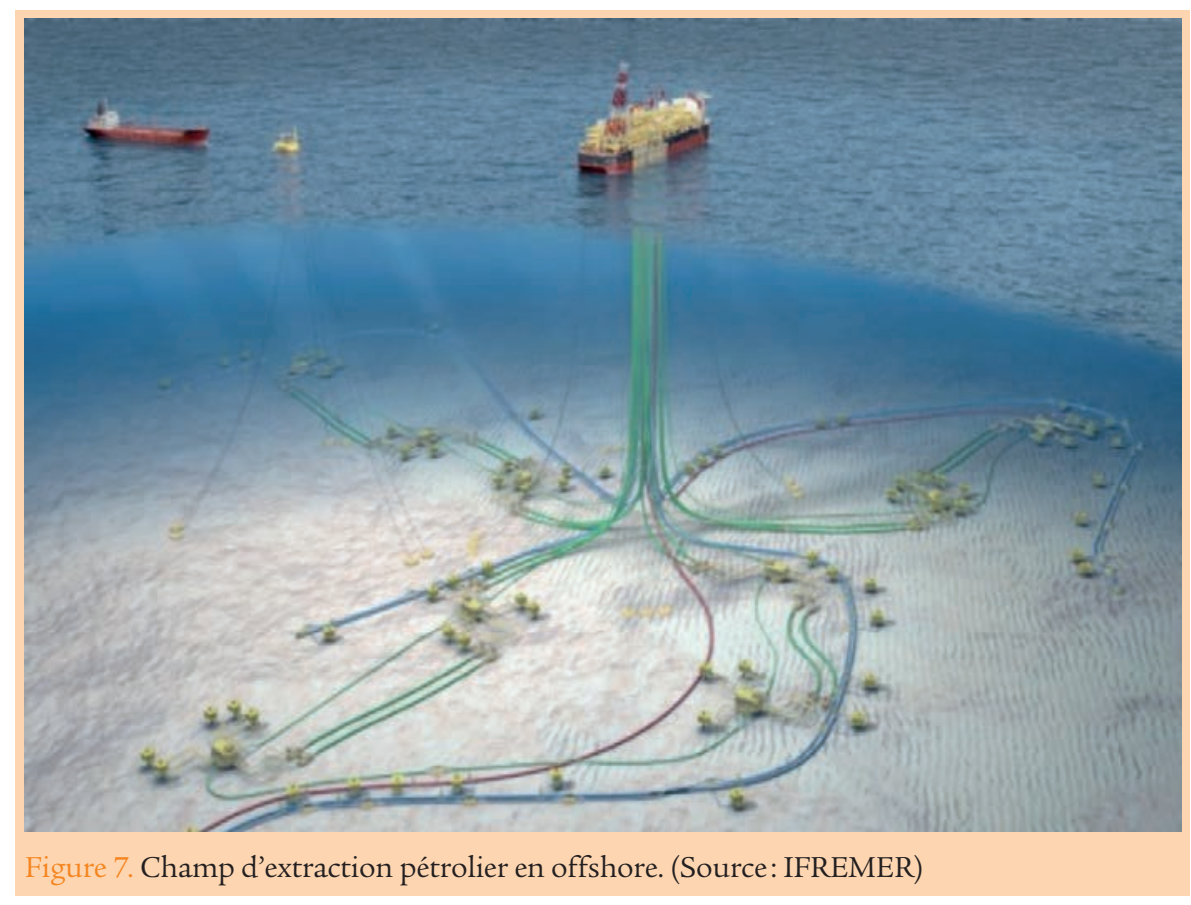

présente de nombreux avantages par rapport aux capteurs traditionnels. En effet, l'atténuation du signal est faible dans les fibres optiques, ce qui permet des mesures déportées à plusieurs dizaines de kilomètres. De plus, elles sont électriquement neutres et insensibles aux bruits électromagnétiques, ce qui peut représenter un atout essentiel, par exemple pour l'instrumentation des centrales électriques. Ensuite, leur diamètre est inférieur au $\mathrm{mm}(0,125 \mathrm{~mm}$ en standard), si bien que le poids et la taille des capteurs limitent au maximum les effets d'inclusion. Le réseau de Bragg faisant une mesure ponctuelle, celui-ci arrive en complément de solutions optiques réparties telle que la mesure par effet Brillouin. Les photographies présentées en figure 5 montrent l'insertion de 6 fibres optiques équipées d'une vingtaine de réseaux de Bragg dans des profilés de composite destinés à la réalisation d'une passerelle de $20 \mathrm{~m}$ de long. Ces réseaux de Bragg permettent de réaliser un suivi du vieillissement des structures ainsi que la mesure de contraintes. Ces travaux ont été réalisés dans le cadre d'un projet collaboratif intitulé DECID II [1].

Les réseaux de Bragg sont aussi utilisés pour le suivi de comportement des chaussées ainsi que de l'enrobé des tarmacs d'aéroports. Les photographies regroupées en figure 6 représentent un conditionnement spécifique de réseau de Bragg (qui est tendu entre deux points) pour insertion dans l'enrobé de tarmac. Des fibres optiques équipées de réseaux ont été insérées dans le tarmac de l'aéroport de Toulouse Blagnac par le Service Technique de l'Aviation Civile (STAC). Ces réseaux de Bragg permettent de mesurer la sollicitation de l'enrobé lors des passages d'aéronefs pendant les phases de décollage et d'atterrissage.

\section{Réseaux de Bragg dans l'offshore}

La fibre optique est présente dans le domaine de l'offshore depuis plusieurs dizaines d'années. Son implantation permet de mesurer des pressions, des températures ou des contraintes. Le réseau de Bragg permet de monitorer différentes structures telles que des conduites d'hydrocarbures.

Par exemple dans le cadre du projet CEPM [2], des conduites en acier ont été équipées de chapelets de 4 réseaux de Bragg sur leur périmètre extérieur. Ces réseaux ont permis de suivre le comportement des conduites sous pression jusqu'à l'endommagement. Ces mesures ont validé la prédiction des calculs sur le comportement des conduites sous pression.

\section{Les réseaux de Bragg dans l'aviation}

Les réseaux de Bragg sont couramment utilisés dans l'aviation. Leur utilisation se fait à la fois lors des qualifications de l'avion, mais aussi de manière définitive lors de l'exploitation de celui-ci. Les photographies en figure 10 représentent une mesure de température réalisée par Airbus autour d'un A380. Trois fibres optiques de $125 \mathrm{~m}$ équipées chacune de 13 réseaux de Bragg

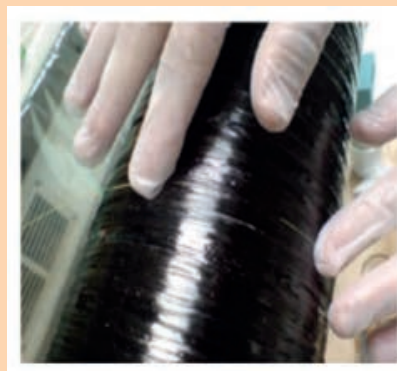

Collage de réseaux de Bragg autour d'une conduite

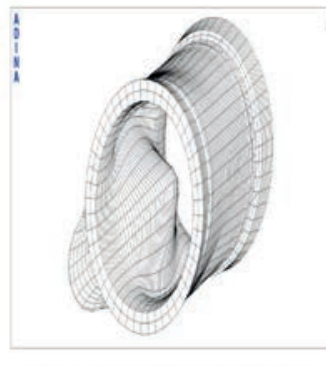

Calcul du comportement d'une conduite

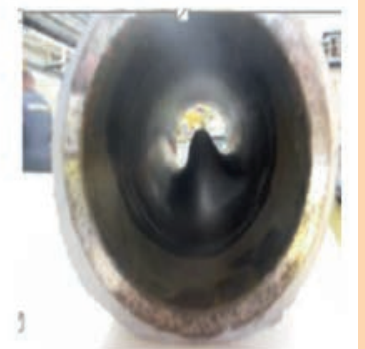

Conduite après endommagement

Figure 8. Conduites en acier équipées de chapelets de 4 réseaux de Bragg sur leur périmètre extérieur et suivi de leur comportement sous pression.

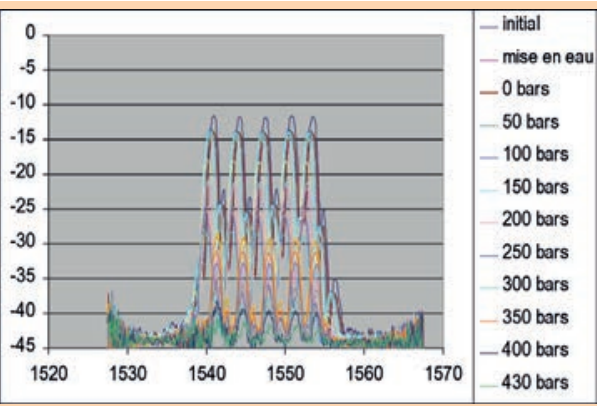

Figure 9. Spectre en réflexion d'une chaîne de 4 réseaux de Bragg équipant une conduite en fonction de la pression. 


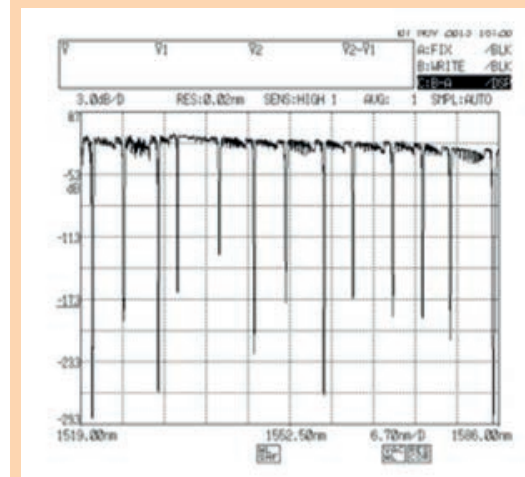

Spectre en transmission de la chaîne de réseaux de Bragg

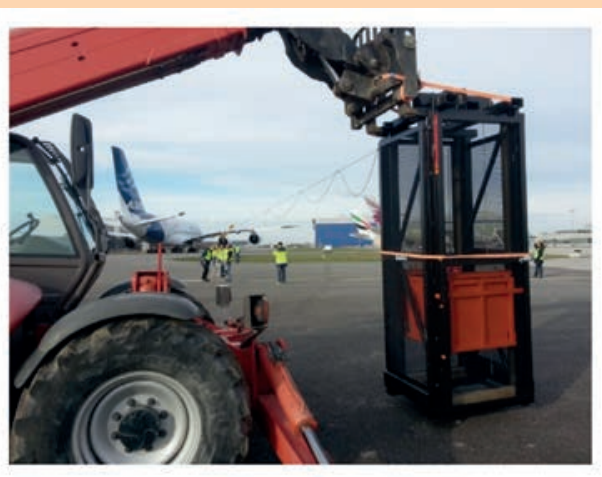

Fibre optique déployée autour de l'A380

Figure 10. Mesure de température autour d'un A380.

ont été utilisées. Ces mesures ont permis de réaliser une cartographie thermique autour de l'avion et ainsi de déterminer son périmètre de sécurité sur les aéroports.

\section{Les réseaux de Bragg dans l'automobile}

Les réseaux de Bragg ainsi que les fibres optiques sont employés depuis quelques décennies dans les domaines cités précédemment. Néanmoins on ne peut pas qualifier ces applications de grand public. Un point de mesure (réseau de Bragg) a un coût de l'ordre de plusieurs dizaines d'euros et une centrale d'acquisition présente un prix de vente qui ne descend pas en dessous de $5000 €$. Ces prix limitent le déploiement à grande échelle de ce type de capteur.

Dans le domaine de l'automobile, les réseaux de Bragg peuvent être utilisés pour la qualification des processus et des véhicules, mais pas encore intégrés dans les véhicules de tourisme. Des études en cours permettent néanmoins d'envisager une réduction importante des coûts par le développement d'interrogateurs bas-coût. La fibre utilisée dans les voitures sert aujourd'hui essentiellement pour l'éclairage d'habitacle (fibre plastique) ou encore pour les systèmes multimédia.

\section{Conclusion}

Nous avons montré quelques exemples d'application des réseaux de Bragg dans le domaine des capteurs. Le nombre d'applications est croissant avec la réduction des coûts. De nombreux travaux restent en cours dans ce domaine afin de faciliter le procédé de fabrication, comme par exemple le développement de gaine transparente aux UV pour éviter de dénuder la fibre, ou la photoinscription dite «Femto» ouvrant de nouveaux champs d'application.

Les premiers réseaux de Bragg ont été implantés il y a maintenant une trentaine d'années. Cette technologie est dorénavant « mature » et autorise son utilisation sans redondance d'une autre technologie.

\section{RÉFÉRENCES}

[1] Projet collaboratif DECID II (démonstrateur en matériaux composites intelligents avec double capacité in-situ du diagnostic de sante structurale en continu par fibres optiques \& capteurs ultrasoniques), partenaires : Ifsttar, DFC, ETPO, Cetim, Université de Rennes I, IXFIBER, IDIL Fibres Optiques. Financement: Région Bretagne

[2] Projet collaboratif CEPEM (système de contrôle intégré de structures composites en mer profonde), partenaires : IFREMER Université P. Sabatier, Armines, Saipem, Structil, IDIL Fibres Optiques. Financement: OSEO

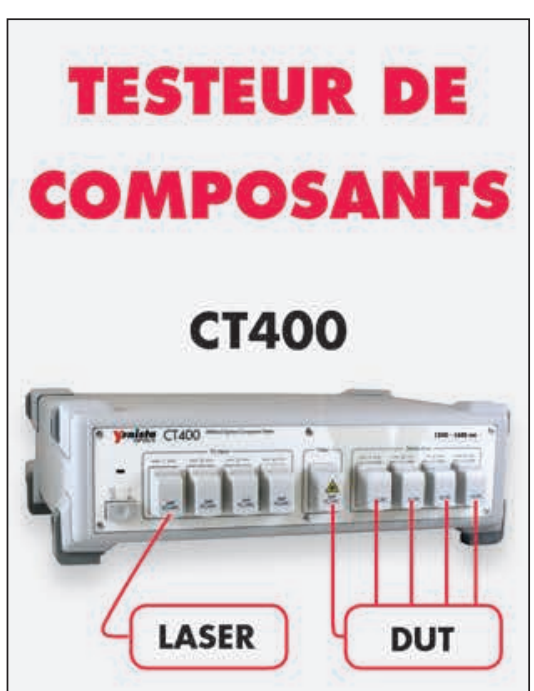

\section{Caractérisation de} Composants Passifs

- Plages de longueur d'ondes :

- SMF : $1240-1680 \mathrm{~nm}$

- PM13 : 1260 - $1360 \mathrm{~nm}$

- PM15 : 1440 - $1640 \mathrm{~nm}$

- Précision : \pm 5 pm

- Résolution: 1 à 250 pm

- Dynamique : $65 \mathrm{~dB}$

- Nombre de lasers : 1 à 4

- Nombre de détecteurs : 1 à 4

- Logiciel client sur PC :

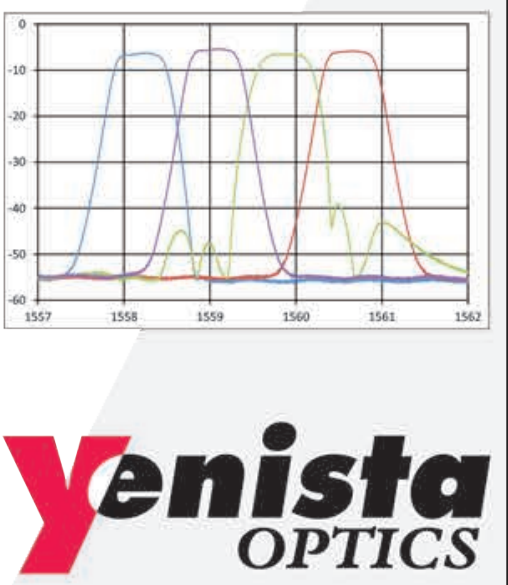

Tél. : +33(0)296483716 sales-emea@yenista.com www.yenista.com 